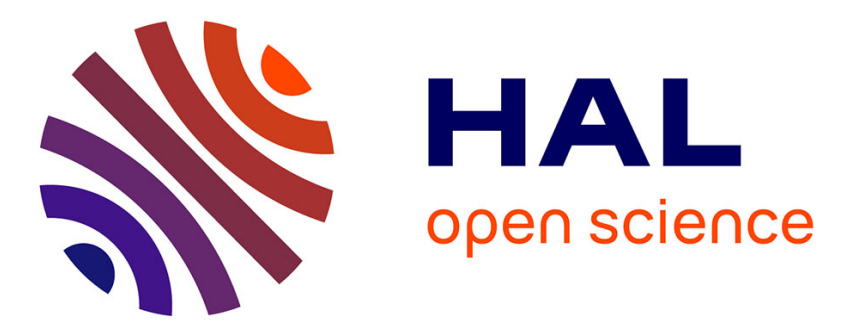

\title{
Coopératives de travailleurs autonomes: du jeu dans les règles au jeu sur les règles, éléments de comparaison Belgique, France et Italie
}

\author{
Marie-Christine Bureau, Antonella Corsani, Bernard Gazier
}

\section{To cite this version:}

Marie-Christine Bureau, Antonella Corsani, Bernard Gazier. Coopératives de travailleurs autonomes: du jeu dans les règles au jeu sur les règles, éléments de comparaison Belgique, France et Italie. Relations Industrielles / Industrial Relations, 2021, 76 (1), pp.3-24. 10.7202/1075571ar . hal-03507548

\author{
HAL Id: hal-03507548 \\ https://hal.science/hal-03507548
}

Submitted on 3 Jan 2022

HAL is a multi-disciplinary open access archive for the deposit and dissemination of scientific research documents, whether they are published or not. The documents may come from teaching and research institutions in France or abroad, or from public or private research centers.
L'archive ouverte pluridisciplinaire HAL, est destinée au dépôt et à la diffusion de documents scientifiques de niveau recherche, publiés ou non, émanant des établissements d'enseignement et de recherche français ou étrangers, des laboratoires publics ou privés. 


\title{
Coopératives de travailleurs autonomes : du jeu dans les règles au jeu sur les règles, éléments de comparaison Belgique, France et Italie
}

\author{
Marie-Christine Bureau, Antonella Corsani et Bernard Gazier*
}

\begin{abstract}
Comment mieux protéger les travailleurs de la «zone grise » entre salariat et travail indépendant qui sont des indépendants économiquement dépendants de leur donneur d'ordre ou qui sont formellement des salariés, mais vont de projet en projet ? Centré sur la situation et l'activité des «I-Pros », des travailleurs autonomes qualifiés et travaillant en solo, cet article explore les stratégies de certains collectifs regroupant ces travailleurs dans trois pays européens : Belgique, France et Italie. Ceux-ci jouent souvent à la fois dans les règles et sur les règles. C'est notamment le cas des entrepreneurs-salariés associés au sein de trois coopératives dont les trajectoires et les logiques d'action sont analysées plus en détail.
\end{abstract}

Mots-clés : travail indépendant, autonomie, coopératives d'activités et d'emploi, salarié contractuel, précarité.

\section{Introduction}

Les formes de travail indépendant qui se développent aujourd'hui en Europe apparaissent très contrastées, de l'essor du «travail de foule » (crowdworking en anglais), ce «travail en miettes » exécuté à la tâche pour le compte de plateformes numériques, à la diffusion de la figure du «I-Pro ». Le I-Pro est un travailleur indépendant et/ou autonome qui travaille en solo dans le secteur des services (à l'exclusion de l'agriculture, de l'artisanat et du commerce) ou qui exerce une activité intellectuelle (Rapelli, 2012). Il s'agit souvent de travailleurs hautement qualifiés qui exercent leur activité en indépendant (freelancer en anglais ou pigiste) ou sous une pluralité de statuts, principalement dans les secteurs de l'informatique, de l'art et du design, de la culture, de la formation, de la communication, de la traduction, du conseil. En raison précisément de leur(s) statut(s) et à l'instar des crowdworkers, les I-pros échappent pour partie aux filets de protection sociale et aux formes de représentation collective instituées dans le cadre de la société salariale. On observe, néanmoins, dans les différents pays d'Europe, des organisations collectives de ces travailleurs qui tentent, soit de faire évoluer les règles en vigueur, soit de les utiliser, voire de les détourner en leur faveur, ce qui fait d'eux de nouveaux acteurs du changement institutionnel.

Les I-Pros, dont le nombre s'est fort accru au cours des années 2010 (Semenza et Pichaut, 2019), peuvent être considérés comme l'une des figures caractéristiques des «zones grises du travail et de d'emploi »(Bureau, Corsani, Giraud et Rey, 2019). Des zones qui

* Marie-Christine Bureau, Chercheure au Laboratoire interdisciplinaire pour la sociologie économique, Conservatoire national des arts et métiers-CNRS, Paris, France (marie-christine.bureau@lecnam.net).

Antonella Corsani, Enseignante-Chercheure, Université Paris 1 Panthéon-Sorbonne, Paris, France (corsani@univ-paris1.fr).

Bernard Gazier, Enseignant-Chercheur émérite, Université Paris 1 Panthéon-Sorbonne, Paris, France (gazier@univ-paris1.fr). 
s'élargissent en même temps que se creuse l'écart entre les institutions et les pratiques. Auparavant, la notion de travail salarié renvoyait à une situation où dépendance et hétéronomie coïncidaient, alors que la figure du travailleur non salarié recouvrait des situations d'autonomie et d'indépendance à la fois. Dans les «zones grises », des travailleurs non assujettis par un lien de subordination juridique peuvent se retrouver en situation d'hétéronomie du fait de leur dépendance économique envers un donneur d'ordre. Symétriquement des figures de travailleurs formellement salariés peuvent bénéficier d'une situation d'autonomie réelle. Indépendance et autonomie ne se recouvrent donc plus (Supiot, 1999).

Depuis les années 1990, l'existence de ces zones a été observée et dénoncée comme génératrice de non-droit, car le droit social a été construit sur la base d'une approche binaire qui oppose le travail salarié et le travail non salarié, le lien de subordination traçant la ligne de partage et donc la répartition des risques et des protections. L'expansion de ces «zones grises » a suscité des réponses institutionnelles variées en termes de protection sociale, sans toutefois parvenir à l'égalité des droits, d'autant que les conditions d'accès aux droits ne sont parfois pas réunies : à titre d'exemple, on peut songer aux difficultés rencontrées en France par les intermittentes du spectacle pour bénéficier du congé maternité (Roux, 2019). Dans ce contexte, certains collectifs d'I-Pros s'engagent dans un «travail instituant » qui vise à la fois la révision des règles de droit pour améliorer leur situation socio-professionnelle, mais aussi la fabrication de nouvelles catégories de pensée, voire de nouveaux imaginaires pour penser les relations de travail. "La contribution à la formation des règles, à leur maintien ou à leurs transformations est consubstantielle à l'action » écrit de Terrsac (2012). En considérant avec Reynaud (1997) que la caractéristique majeure de l'acteur social est sa capacité de construire des règles sociales et d'y consentir, nous choisissons de centrer notre analyse sur l'élaboration des règles qui encadrent l'existence des I-Pros. Cette perspective est d'autant plus intéressante que les I-Pros constituent de nouveaux acteurs, encore faiblement organisés, dans le champ des relations professionnelles. Néanmoins, la notion de travail instituant, formulée initialement par Castoriadis (1975) et reprise par Nicolas Le-Strat (2015), déborde d'une certaine façon la négociation des règles pour embrasser un ensemble plus vaste de réalités institutionnelles, à commencer par les catégories qui nous servent à désigner les situations, penser le monde, voire imaginer le futur. En conjuguant la notion de travail instituant avec les apports de Mahoney et Thelen (2010) pour penser le rôle du jeu sur les règles dans les transformations graduelles, nous adoptons une approche pragmatique du changement institutionnel, tout en reconnaissant le pouvoir de l'imaginaire dans la «fabrique instituante » (Bureau et Corsani, 2019).

Notre réflexion sera menée selon trois échelles distinctes, partant des contextes nationaux pour se focaliser sur les organisations et les jeux d'acteurs. Nous prenons ici appui en partie sur les matériaux réunis à l'occasion du projet européen I-WIRE ${ }^{1}$ (Independent Workers and Industrial Relations, 2016-2018) dont le but était de repérer les besoins de protection juridique et sociale des I-Pros dans neuf pays d'Europe, ainsi que les formes d'organisation et de dialogue social qui permettent ou non d'y répondre (Semenza et Pichault, 2019). L'une des phases du projet consistait à identifier, dans chaque pays, des collectifs pertinents menant une action en faveur de ces travailleurs, et à étudier plus précisément leurs modes d'action. Parmi ces collectifs, deux ont retenu tout particulièrement notre attention : SMart en Belgique et Coopaname en France. Nous avons été intéressés aussi par un troisième collectif qui n'avait pas été analysé lors de la réalisation du projet I-WIRE, Doc Servizi en Italie. Ces trois collectifs prennent une forme originale : il s'agit, en effet, de coopératives de 
travailleurs autonomes. Dans un premier temps, nous caractériserons brièvement la situation des nouvelles formes de travail au regard des institutions du travail (salarié et non salarié) et des formes syndicales existant dans les trois pays. Puis, nous nous intéresserons à la diversité des modalités d'action des collectifs identifiés dans le cadre du projet I-WIRE, mettant en exergue la spécificité de la logique coopérative. Enfin, nous examinerons la situation et la trajectoire des trois coopératives repérées. Outre les investigations menées dans le cadre du projet I-Wire, nous mobilisons pour ce faire les connaissances acquises depuis plusieurs années de recherche sur les Coopératives d'activité et d'emploi (CAE) en France, ainsi que les apports d'un travail doctoral comparant Coopaname et Doc Servizi (Martinelli, 2016).

L'originalité de notre apport repose sur la mise en évidence d'une nouvelle logique d'action collective des I-Pros, la logique coopérative, explicitée à travers l'histoire croisée de trois expérimentations. Notre propos est de montrer comment des initiatives nées dans des contextes institutionnels différents tendent, de fait, à converger, par le jeu de leurs influences réciproques, dans l'invention de formes institutionnelles et organisationnelles similaires.

\footnotetext{
Encadré méthodologique : les matériaux de recherche mobilisés

\section{1- Participation française à l'enquête européenne I-WIRE}

- organisation systématique des données qualitatives et quantitatives existantes selon une méthodologie commune aux différentes équipes européennes

- des entretiens semi-directifs avec une dizaine de représentants de différentes organisations de travailleurs autonomes et/ou indépendants en France

- organisation d'une rencontre au Conservatoire national des Arts et Métiers entre cinq organisations de travailleurs autonomes en France

- une enquête en ligne auprès des I-Pros, sur la base d'un questionnaire commun aux neuf pays du projet I-Wire : 158 répondants en France, contactés par le biais de plusieurs réseaux d'I-Pros

- analyse transversale des formes d'action collective des travailleurs autonomes FranceBelgique-Italie

2/ Observation participante (2011- 2018) et Enquête à Coopaname dans le cadre d'une Recherche-Action participative (2014-2017)

- Rencontres et entretiens avec des membres et la direction de SMArt (Belgique)

- Participation Observation des rencontres en vue de la transformation de SMArt en coopérative
} 
- Observation de deux rencontres entre Coopaname (France) et DOC Servizi (Italie)

- Observation des rencontres entre Coopaname et SMArt 


\section{Statuts et protections du travail indépendant et/ou autonome : trois situations nationales contrastées}

Les configurations légales et institutionnelles au sein desquelles s'exerce le travail indépendant, notamment celui du groupe des indépendants qualifiés, sont très diverses. Pardelà la variété des arrangements nationaux, on observe l'existence d'« équivalents fonctionnels » : des institutions ou des règles différentes produisant des résultats comparables. C'est ainsi que la catégorie des artistes obtient dans la plupart des pays une protection spécifique, mais selon des modalités différentes allant d'avantages fiscaux/sociaux à des statuts dédiés.

Le statut même d'indépendant se définit la plupart du temps négativement : le travailleur indépendant est le travailleur qui n'est pas salarié : en France, ni salarié ni travailleur agricole; en Belgique, le travailleur indépendant est celui qui ne bénéficie pas d'un contrat de louage; en Italie, l'opposition est plus précise, le Code civil distinguant le salarié, auquel s'applique l'obligation de moyens et la fourniture de travail durant un laps de temps déterminé, et le travailleur indépendant auquel s'applique l'obligation de résultat auprès de clients.

Dans tous les cas, la préoccupation du législateur a longtemps été de distinguer clairement les deux situations, afin de partager les risques et les responsabilités et attribuer des protections. Toutefois, en dépit de cette ligne de démarcation, on a vu que se sont multipliées dans les trois pays (ainsi que dans bien d'autres) des situations intermédiaires, des «zones grises » où les emplois juxtaposent des traits des deux statuts, avec souvent le cumul de l'indépendance formelle et de pratiques de dépendance caractéristiques de la subordination salariale. Il en résulte des formes renouvelées de précarité. Celles-ci, générées notamment par la discontinuité des engagements et des contrats, ainsi que par la dépendance envers un ou plusieurs donneur(s) d'ordre, diffèrent des situations vécues par les indépendants traditionnels que sont les artisans ou les professions libérales dont l'activité et la sécurité reposent sur une clientèle qu'il s'agit de fidéliser et d'élargir.

Face à cette nouvelle précarité, un premier contraste entre les trois pays est celui de l'importance des partenaires sociaux et du dialogue social. Une grande différence existe entre la Belgique, dont les syndicats agissent principalement dans une logique de syndicalisme de service, et l'Italie et la France, dont les syndicats promeuvent une logique d'action revendicative militante. On observe en Belgique des syndicats aux effectifs très nombreux (50,3\% des salariés en 2018 selon l'OCDE) et aux attributions vastes, comprenant par exemple la cogestion de l'assurance-chômage, ce pays se rapproche ainsi du «modèle nordique ». Si l'on peut qualifier la France et l'Italie de «modèles méditerranéens », avec des syndicats de militants aux effectifs moindres dans un contexte d'érosion durable, le contraste est fort ici encore entre les deux pays. En Italie, les syndicats conservent des effectifs nombreux $(34,4 \%$ des salariés en 2018$)$ et sont associés à la gestion d'importantes garanties sur l'emploi et la retraite dans le secteur industriel. Ils participent, dans le cadre d'une forte régionalisation, à diverses versions du dialogue social territorial, allant de la fixation des salaires à la protection de certaines catégories de travailleurs et à la gestion d'avantages sociaux. En France, avec un taux de syndicalisation qui est l'un des plus faibles d'Europe $(8,8$ $\%$ des salariés en 2018), mais un taux de couverture des accords proche de 100\%, l'influence des syndicats, eux-mêmes divers et clivés entre participation systématique aux négociations et pressions protestataires, s'apparente à celle de partis politiques. La France et l'Italie ont en commun un fort dualisme du marché du travail, une partie des emplois étant bien protégée et 
l'autre, la plupart du temps hors de la représentation syndicale, correspondant à un segment «secondaire » et instable du marché du travail. Ce dualisme est comparativement moins prononcé en Belgique.

L'appareil de protection sociale est extrêmement développé en Belgique et en France, et à un moindre degré en Italie. Croisant les repérages de ces deux échelles, on peut donc opposer un pays quasi-« nordique », la Belgique, dont la socialisation est très poussée qu'il s'agisse de la solidarité ou du dialogue social, à deux versions très différentes de pays typiques d'Europe du Sud, marqués par divers dualismes, certaines catégories de travailleurs étant bien protégées et d'autres non. La France compense partiellement ses dualismes par l'action d'un appareil redistributif et d'une protection sociale forts, cependant que l'Italie demeure plus fragmentée, des syndicats localement puissants et représentatifs étant à même de compenser dans certains secteurs et territoires, mais ne pouvant agir partout.

Il en est résulté, en ce qui concerne le travail indépendant, des évolutions selon des modalités et des rythmes très différents. Un premier contraste est celui de l'importance quantitative du travail indépendant. Pour 2016, l'OCDE (qui définit l'indépendant comme un travailleur non salarié, n'ayant pas lui-même de salarié) donne, en pourcentage du total des travailleurs : $23,9 \%$ pour l'Italie, $14,8 \%$ pour la Belgique et 11,8\% pour la France. Ces chiffres ne doivent pas cacher l'extrême hétérogénéité des situations, qui va des professions réglementées et solidement encadrées aux statuts les moins protégés, tels que les diverses catégories de travailleurs indépendants (freelance en anglais) dans les trois pays considérés.

Le très fort développement du travail non salarié en Italie va de pair avec d'importantes interventions légales visant à le limiter et le sécuriser. Deux décrets de 2015 et 2017 ont largement réformé le traitement des travailleurs indépendants, avec l'ambition de marquer un nouveau départ. Appartenant tous deux à l'ensemble des réformes du marché du travail regroupées sous l'intitulé de «Jobs Act», le premier réforme le contrat de travail, alors que le second précise le statut de l'auto-emploi. Il s'agit, d'abord, de limiter les abus auxquels avaient donné lieu deux contrats de travail hybrides instaurant une situation intermédiaire, celle de la «para-subordination »: le « cococo» (Contratto di collaborazione coordinata et continuativa, introduit en 1997) et le « cocopro » (Contratto a progetto, introduit en 2003). Il s'agit ensuite de rendre possible l'accès à de nouveaux droits pour les travailleurs concernés, notamment en ce qui concerne la maternité.

En Belgique, l'activité législative est bien moins importante. Une tentative de créer un nouveau statut hybride a eu lieu en 2017, mais a tourné court. Face à une fiscalité parafiscalité lourde et complexe, prévoyant notamment des versements anticipés -, les changements récents ont porté sur des allégements fiscaux pour les travailleurs indépendants : en dessous d'un seuil de revenu très bas, 5000 Euros, le taux d'imposition est de $10 \%$ alors qu'il reste $33 \%$ au-dessus de ce seuil.

En France, après la création du statut «d'auto-entrepreneur » en 2009 — statut aux contraintes sociales allégées, mais ouvrant des droits restreints - ce sont des réformes affectant le salariat standard (assouplissant les modalités de rupture du contrat) et non standard (taxation accrue des Contrats à Durée déterminée de courte durée) qui ont dominé l'agenda réformateur des années 2010. Un changement important est la décision, en 2016, d'intégrer le RSI, régime de protection sociale des indépendants, au régime général des salariés, entérinant la volonté de rapprocher les conditions de gestion et les garanties de celles du statut salarié. 
Les comportements des acteurs face à cette mosaïque de contraintes et au développement de situations à la fois précaires et peu visibles ont souvent consisté à tenter de ramener au moins certaines d'entre elles au salariat. Il peut s'agir de requalification par un juge, d'assimilation ou encore de création statutaire originale, tels les intermittents du spectacle en France, qui vont de projet en projet mais, au-delà d'un nombre d'heures annuelles déclarées, bénéficient d'une protection sociale comparable à celle des salariés.

Complémentaires de ces efforts de sécurisation, diverses formes de représentation et d'expression des indépendants, via des syndicats ou des quasi-syndicats, se sont développées dans les trois pays. On y observe aussi l'apparition de tiers venant s'interposer entre le client et le travailleur et visant à sécuriser la carrière de leurs adhérents. De tels tiers peuvent être très variés, certains sont des entreprises capitalistes, telles la plupart des sociétés de portage salarial - auxquelles l'indépendant confie ses recettes, à charge pour elles de payer les cotisations sociales afin de lui apporter les protections du salariat au prorata de son chiffre d'affaires. D'autres, comme les Coopératives d'activité et d'emploi (cf. infra), relèvent de l'économie sociale et solidaire.

Il résulte de ces paysages très divers que souvent les nouveaux travailleurs indépendants, à la fois qualifiés et relevant de professions peu ou mal normées, ne sont pas « dans les tuyaux » normaux de la protection sociale et de la législation de l'emploi. Percevant des revenus irréguliers et souvent faibles en moyenne, disposant de peu, voire pas de protection en ce qui concerne les accidents du travail, bénéficiant de protections minimales en matière de santé et accumulant peu de droits à la retraite, ces travailleurs souffrent aussi d'un déficit de représentation et d'expression collectives. Leur inconfort est à la fois objectif et subjectif, certains peuvent revendiquer la position d'indépendant y compris dans des contextes de dépendance visible. Comme l'a noté, en janvier 2020, une sénatrice française examinant une proposition de loi visant à sécuriser les travailleurs des plateformes numériques : "On compte (...) à ce jour peu de demandes de requalification pour les travailleurs des plateformes, ceuxci ayant à court terme une préférence pour le statut d'indépendant qui leur garantit une souplesse dans l'organisation de leur temps de travail et une rémunération immédiate plus élevée. » (Grelet-Certenais, 2020 : 11).

Cet inconfort, ainsi que les capacités de compréhension et de mobilisation qui sont grandes chez les travailleurs qualifiés opérant dans les secteurs émergents, expliquent sans doute la créativité dont ils peuvent faire montre non seulement pour conquérir une meilleure protection sociale, mais aussi pour affermir leur autonomie et mieux sécuriser les conditions concrètes d'exercice de leurs activités à travers l'action collective.

\section{Acteurs, règles et changement institutionnel}

Mori et Koene (2019) insistent sur le fait que l'émergence de collectifs visant à représenter les travailleurs autonomes est un phénomène récent des deux dernières décennies, même si, dans certains secteurs professionnels réglementés, il existe des formes beaucoup plus anciennes, telles que l'AJP, association de journalistes professionnels née en Belgique dès la fin du $19^{\text {ième }}$ siècle, soit en1886. Certains syndicats ont compris la nécessité d'ouvrir leurs portes aux I-Pros et de leur offrir des services adaptés, comme la Fédération Culture F3C de la CFDT en France ou la CISL (Confédération italienne des syndicats de travailleurs) avec la création de vIVAce (Association italienne des Indépendants et de membres de professions libérales). Mais le développement d'organisations collectives se fait le plus souvent selon une logique ascendante ou de bas en haut (bottom-up en anglais), en dehors des 
organisations instituées et à l'initiative des personnes concernées, comme dans le cas d'ACTA (Association des Consultants du Tertiaire Avancé), née en Italie du regroupement d'une vingtaine de professionnels indépendants ou pigistes, ou encore SMArt (Société mutuelle pour Artistes), fondée en Belgique par le manager d'un groupe de musique et un ingénieur, et enfin les Coopératives françaises d'Activités et d'Emploi, inventées dans les années 1990 par des intervenantes sociales.

Pour caractériser les modes d'action de ces nouveaux collectifs, nous utiliserons, en la complétant, la typologie proposée par D'Amours (2010) analysant la situation au Québec. Celle-ci distingue d'abord plusieurs niveaux d'action collective : le niveau organisationnel (services rendus aux membres); le niveau institutionnel (appel à l'État, revendications, négociation des conditions de travail et de rémunération avec les donneurs d'ordre); le niveau d'action sur les rapports sociaux. Elle identifie alors cinq logiques d'action collective des travailleurs indépendants: la logique entrepreneuriale, la logique syndicale classique, la logique syndicale élargie, la logique professionnelle et la logique des normes minimales universelles. La logique entrepreneuriale vise essentiellement à défendre les intérêts des micro-entreprises en améliorant les règles fiscales et sociales qui leur sont appliquées. La logique syndicale classique s'efforce d'étendre à un maximum de travailleurs la protection des lois du travail, y compris le droit à la syndicalisation, tandis que la logique syndicale élargie se présente comme une sorte $\mathrm{d}$ ' "amalgame des deux premières »: elle est, par exemple, incarnée au Québec par les associations d'artistes qui revendiquent tant une identité de travailleurs indépendants qu'un droit de négociation collective. La logique professionnelle est celle des ordres professionnels qui, « en contrepartie de règles visant à protéger le public, créent des marchés du travail protégés de la concurrence par des barrières à l'entrée institutionnalisées et par le monopole sur la livraison de certains biens ou services » (D'Amours, 2010 : 269). Enfin, la logique des normes minimales universelles concerne surtout les travailleurs vulnérables, catégorie bien différente de celle des I-Pros, pour lesquels est revendiquée une présomption de salariat. C'est le cas notamment des livreurs sur les plateformes.

Si nous suivons les choix effectués par les équipes d'I-WIRE dans les trois pays pour identifier des organisations regroupant des «I-Pros », la grille de D'Amours permet de rendre compte de la plupart des cas étudiés. La Fédération française des «auto-entrepreneurs » incarne la logique entrepreneuriale, tandis que les registres d'action de la CGT, de vIVAce et des CLAP (Chambres du Travail Autonome et Précaire, créés en Italie) relèvent des logiques syndicale et syndicale élargie. L'association belge des journalistes (AJP), tout comme l'Alliance française des Designers (AFD), relèvent d'une logique professionnelle, à condition d'élargir la notion au-delà de l'établissement de barrières à l'entrée, pour désigner un mode d'action attentif à construire un lien permanent entre qualité des services et qualité du travail (déontologie, régulation des tarifs pratiqués, formation continue, etc.). Par rapport à la grille initiale, nous devons toutefois introduire une nouvelle logique d'action qui en était absente : la logique coopérative. Cette logique est celle de Coopaname en France, de SMArt en Belgique et de Doc Servizi en Italie ${ }^{2}$. La logique coopérative conjugue différents niveaux de l'action collective, notamment les niveaux institutionnel et organisationnel, et agence de manière singulière les logiques entrepreneuriale, syndicale et professionnelle. Mais sa particularité réside essentiellement dans le fait d'associer la garantie d'accès aux droits sociaux du salariat à la mutualisation des ressources, avec pour horizon la volonté d'inventer et de consolider une nouvelle forme d'entreprise coopérative constituée de travailleurs autonomes. Le choix du statut coopératif pour cette nouvelle forme d'entreprise n'est pas sans signification spécifique, 
car le statut coopératif implique aussi une forme de démocratie d'entreprise (une personne, une voix) et une affectation des résultats qui privilégie le collectif, comme collectif de travail (distribution aux salariés) ou comme entreprise (investissement).

En reprenant la dimension créatrice de la régulation chère à Reynaud (1997), nous nous intéressons ici à la façon dont les différents acteurs collectifs identifiés dans les trois pays agissent pour faire évoluer, voire pour transformer les règles du jeu, devenant ainsi parties prenantes du changement institutionnel. Les logiques d'action diffèrent entre elles a priori par la nature des règles sur lesquelles elles entendent agir. Syndicats et «quasi-unions » s'efforcent, en premier lieu, d'améliorer les conditions de travail et les droits sociaux des indépendants vus avant tout comme des travailleurs, indépendamment de leur statut juridique. En revanche, les micro-entrepreneurs s'organisent pour peser sur les règles sociales et fiscales spécifiques aux micro-entreprises, ou pour tenter de rééquilibrer les rapports de force qu'ils subissent sur le marché des services face à leurs donneurs d'ordre. Les associations professionnelles visent, quant à elles, à réguler de façon concomitante marché des services et marché du travail. De même, la logique coopérative met directement en question la distinction inscrite au cœur du droit du travail entre salariat et indépendance et elle agit, de fait, à la fois sur les règles du travail et celles du marché.

Si l'on regarde de plus près les actions menées par les acteurs collectifs, on remarque que les logiques sont largement entremêlées. Les actions visent à faire évoluer, à des degrés divers, un large éventail de règles, parmi lesquelles on peut distinguer :

- Les règles fiscales et sociales de la micro-entreprise (droit des sociétés)

- Les règles d'encadrement des contrats de sous-traitance ou prestations de service (droit commercial)

- Les règles de rémunération et de conditions de travail (droit du travail)

- Les règles d'accès aux droits sociaux (droit social)

- Les règles d'accès à l'exercice des activités professionnelles (lois de réglementation des professions)

- Les règles éthiques de comportement (chartes)

Le Tableau 1 donne à voir, pour chacun des collectifs identifiés dans le projet i-WIRE, des exemples de mode d'action selon la nature des règles qu'il s'agit de faire évoluer.

\section{(Insérer le Tableau 1 ici)}

Ce tableau montre que la plupart des acteurs collectifs identifiés tentent d'agir simultanément sur plusieurs règles de droit, relevant pour certaines du droit social, pour d'autres du Code du commerce. De fait, ils prennent acte des interdépendances entre les déséquilibres de marché (contrats abusifs, etc.) et les conditions de travail des prestataires, qu'ils soient formellement salariés ou indépendants (Frison-Roche, 2002). En revanche, l'action sur les règles éthiques reste davantage circonscrite aux acteurs professionnels (déontologie) ou coopératifs (démocratie interne). Logique professionnelle et logique syndicale élargie peuvent fort bien aller de pair comme l'avait montré Dupuy (2014) et comme en témoigne la campagne « Pigiste pas pigeon » (Mori et Koene, 2019:192) engagée par l'AJP pour attirer l'attention du grand public sur les conditions dégradées de travail et de rémunération des journalistes indépendants. 
La logique coopérative se distingue tout particulièrement par sa capacité à intervenir sur une palette étendue de règles concernant à la fois le droit du travail, le droit social, le droit commercial et les normes éthiques. L'étendue de son champ d'action explique notre choix de mettre désormais la focale sur SmartBe, Coopaname et Doc Servizi.

\section{Trois coopératives dans les zones grises : singularités et convergence}

Les trois coopératives sont de tailles très différentes. Selon le Rapport d'activités 2019, à la fin de l'année précédente, SMArt en Belgique comptait plus de $90^{\circ} 000$ personnes inscrites, tous motifs confondus (être salarié, suivre une formation, etc.) et le nombre de ses salariés dépasse $20^{\circ} 000$. À la même date, Doc Servizi regroupait $6^{\circ} 388$ membres associés, alors qu'à Coopaname, on dénombrait quelques 800 entrepreneurs, dont environ 300 membres associés. Ces trois coopératives de travailleurs autonomes ont «bricolé », de manière singulière et à partir de contextes institutionnels différents, les règles du droit social de sorte à garantir à leurs membres l'accès à la protection sociale des travailleurs salariés.

L'histoire de ces trois coopératives commence dans les années 1990. Elles ont été créées pour répondre aux besoins de travailleurs formellement et, parfois aussi, réellement économiquement indépendants en situation de précarité conçue comme discontinuité et imprévisibilité. Cependant, comme on a pu le voir, les contextes institutionnels nationaux sont hétérogènes. Il s'en suit que les besoins, bien que similaires, diffèrent et les solutions institutionnelles possibles pour les satisfaire aussi. Par-delà ces hétérogénéités, les trois expériences conduisent toutes à la construction d'une figure nouvelle dans le monde du travail, celle de l'entrepreneur-salarié-associé, bien qu'elle ne soit pas instituée comme telle partout.

\section{Des histoires singulières}

SMArt, «Société mutuelle pour Artistes » a d'abord été une « entreprise sociale », créée en 1998 en Belgique, sous le statut d'ASBL (association sans but lucratif). À l'origine de la création de SMArt, il y a deux personnes, Pierre Burnotte et Julek Jurowicz, qui l'ont dirigée jusqu'en 2014. L'un comme l'autre étaient proches du monde du spectacle et sensibles aux problèmes des artistes. En 1990, ils avaient constitué une première association, «Les Passions Unies », dont la fonction était de fournir aux adhérents - pour la plupart des artistes du secteur des musiques émergentes — des services de secrétariat social. Plus tard, l'association a assuré aussi le suivi administratif des productions de spectacles et des productions discographiques, ainsi que le suivi comptable, financier et fiscal et, enfin, la négociation des contrats. Jusque-là, l'action de l'association relevait strictement du premier niveau défini par D'Amours (2010) : il s'agissait d'une organisation offrant des services à ses membres suivant une logique à dominante professionnelle ${ }^{3}$. Au fil du temps, les besoins des artistes se précisent : il est apparu nécessaire d'entamer une action sur le cadre législatif afin que les artistes puissent exercer leur activité dans de meilleures conditions. En 1998, la création de SMArt entend apporter des réponses aux demandes des artistes. Ses missions sont d'assurer la gestion des contrats, d'apporter de l'information et de défendre les membres devant les administrations et tribunaux, «de manière à aboutir à une jurisprudence plus favorable aux professionnels de la création au sens le plus large. » (Graceffa, 2016 : 97). Dès 2000, SMArt a participé aux travaux de la Plateforme nationale des Artistes, puis, en 2001, aux négociations qui ont abouti un an plus tard à l'adoption de la Loi sur le Statut social de l'artiste. L'artiste qui, sans être lié à un donneur d'ordre par un contrat de travail, lui fournit des prestations artistiques ou des œuvres et qui reçoit en contrepartie une rémunération, bénéficie du statut 
d' ' entrepreneur quasi-salarié ». C'est à ce titre qu'il acquiert le droit à la sécurité sociale des salariés. À partir de l'institution du statut de l'artiste, SMArt joue le rôle de tiers-payant entre le prestataire et son donneur d'ordre. Son modèle économique se base essentiellement sur le pourcentage prélevé sur le chiffre d'affaires réalisé par les membres.

Autre fonction de SMArt, la mutualisation des risques. En 2001, SMArt avait mis en place le Fonds de garantie salariale, dont la fonction est double : faire des avances de salaire dans l'attente du règlement de la facture, mais aussi garantir le paiement en cas de faillite du donneur d'ordre. Cette fonction permet une démocratisation de l'accès aux activités nonsubordonnées, dans la mesure où le Fonds neutralise les risques propres de l'entrepreneuriat (Graceffa, 2016). Il est à remarquer que le Fonds est alimenté par les « donneurs d'ordre » qui contribuent à hauteur de $2 \%$ du montant du contrat. En 2014, SMArt amorce un processus de métamorphose en se transformant d'association en coopérative. Nous y reviendrons.

L'histoire de Doc Servizi, en Italie, est très différente. Doc Servizi est née d'emblée sous forme coopérative, il s'agit plus précisément d'une Coopérative à dominante mutualiste (Cooperative a mutualità prevalente), c'est-à-dire une coopérative dont l'activité est réalisée par ses membres et destinée essentiellement à ces mêmes membres. Doc Servizi opère essentiellement dans le champ de l'art et de la culture et regroupe tous les métiers du spectacle vivant et enregistré.

Elle a été créée à Vérone en 1990, à l'initiative de musiciens, dans le but de dé-précariser les artistes, de garantir leur autonomie, tout en leur assurant en même temps les avantages du salariat. Sa logique d'action relève d'abord d'une logique syndicale élargie. En Italie, les artistes se situent dans un angle mort du droit du travail et la plupart d'entre eux, notamment les artistes du spectacle, travaillent en dehors de tout rapport légal de travail. Pour atteindre ses objectifs, Doc Servizi utilise, en en détournant l'usage, le contrat de travail intermittent, appelé aussi le «Call Jobs », et produit des services ad hoc pour les artistes. Il s'agit ainsi de « détournement institutionnel »: les institutions existantes sont bricolées pour les adapter aux besoins spécifiques, sans que cela relève pour autant de l'illégalité.

Le contrat de travail intermittent est un contrat de travail subordonné par lequel le travailleur se met à la disposition de l'employeur pour effectuer des prestations de travail de caractère discontinu ou intermittent définies par la convention collective nationale ou territoriale, ou bien pour des périodes pré-fixées sur la semaine, le mois ou l'année. On peut véritablement parler de «détournement », car, en réalité, comme dans les sociétés de portage salarial en France, les artistes salariés associés de Doc Servizi ne sont pas tenus par un lien de subordination. Chaque fois qu'ils reçoivent une commande d'un donneur d'ordre, le contrat de travail intermittent s'active.

Tout comme SMArt, et, on le verra, Coopaname, la coopérative italienne offre d'abord un service d'administration et de gestion comptable et fiscale. Mais elle va plus loin : Doc Servizi se constitue en organisateur d'événements de sorte à créer des opportunités de travail pour ses membres. Troisième catégorie de services : des services à l'activité qu'on pourrait qualifier d'indirects. La formation en premier lieu, notamment la formation à la sécurité au travail. Plus originaux sont les services d'agence de voyage et d'édition musicale. L'agence de voyage a comme fonction de permettre aux associés de pouvoir prendre des vacances au moindre coût, mais elle vise aussi à accompagner le public là où les artistes se produisent. Le développement de ces activités s'est fait par création d' "entreprises en démarrage » (startups en anglais) qui font partie aujourd'hui du réseau Doc Servizi. L'activité de promotion des activités de ses membres est un trait qui distingue nettement Doc Servizi de Coopaname. En 
tant qu'éditeur de musique par exemple, elle établit des contrats d'auteur très favorables à ses membres: les droits perçus par l'éditeur s'épuisent dès que les coûts de l'édition sont couverts ${ }^{4}$. Le modèle économique de Doc Servizi ne fonctionne pas seulement sur la base des taux prélevés sur le chiffre d'affaires, mais aussi sur une activité économique établie en complément de l'activité de ses membres.

Coopaname, à la différence de SMArt et de Doc Servizi, n'est pas née dans les milieux artistiques. Et bien qu'elle soit la plus grande Coopérative d'activité et d'emploi de France, elle reste, comme déjà souligné, de taille relativement modeste, si on la compare à SMArt en Belgique ou à Doc Servizi en Italie. Le concept qui la fonde, le concept de Coopérative d'activité et d'emploi (CAE), date du milieu des années 1990. Il a été inventé par Elisabeth Bost qui créa la première CAE à Lyon, Cap Services, avec l'idée d'une entreprise coopérative qui exerce à la fois des fonctions d'insertion par l'économique, d'accompagnement à la création d'entreprise et de sécurisation des parcours. Tout comme une entreprise de portage salarial, la CAE transforme le chiffre d'affaires net (ou les honoraires ou autre forme de rémunération) en salaire. Ce faisant, elle permet à des travailleurs autonomes d'accéder au statut de salarié et, par conséquent, aux droits sociaux du salariat. La synergie entre ces différentes fonctions se manifeste particulièrement en 2004, avec la création, incitée par Elizabeth Bost elle-même, de Coopaname, la première CAE parisienne (Bost, 2011) : avec Coopaname, la CAE ne se conçoit plus comme un fournisseur de services. Il ne s'agit plus seulement de sécuriser les parcours des créateurs d'entreprise, mais d'imaginer et réaliser une entreprise coopérative nouvelle, une entreprise non hiérarchique dont le collectif de travail se compose de travailleurs autonomes et interdépendants au sein de la structure. L'action collective de Coopaname vise à transformer les rapports sociaux de production et de coopération. L'utopie concrète est celle d'une entreprise sans patrons (Poncin, 2004).

En outre, Coopaname est ouverte à tout projet, dans une large palette de métiers. En d'autres termes, elle vise une démocratisation de l'accès à des formes de travail autonome et l'extension à un maximum de personnes du salariat entendu comme protection solidaire interprofessionnelle. La logique de son action est essentiellement politique : il s'agit de réinventer l'entreprise, en travaillant les relations de pouvoir qui se déploient en son sein.

\section{Développements et convergences}

La logique d'action commune à SMArt, Doc Servizi et Coopaname est de s'adapter aux institutions existantes, de les bricoler, les détourner, les utiliser comme levier pour finalement créer quelque chose de nouveau qui réponde mieux aux besoins spécifiques de certaines catégories de travailleurs.

Ce mode d'action expose parfois les coopératives à des risques, assumés de manière consciente. Par exemple, SMArt a été accusée de fraude fiscale, du fait d'avoir mis en place un système pour compenser la mise à disposition des outils de production matériels dont les « salariés » s'équipent à leur charge ${ }^{5}$. Ces dispositifs visant à indemniser les membres ont été considérés par l'administration fiscale comme frauduleux, dans la mesure où l'argent attribué à l'investissement était soustrait du revenu soumis à la cotisation et à l'impôt.

SMArt a aussi réalisé un effort technologique très important pour automatiser les actes de gestion administrative et garantir, grâce à des logiciels très performants, l'autonomie de la personne vis-à-vis de la structure. La mutualisation des outils présente un avantage économique pour les personnes, car elle permet de réduire les coûts supportés individuellement par chacun. SMArt a connu une très forte croissance, tant en Belgique qu'en 
Europe. Entre 2008 et 2018 , plus de $61^{\circ} 000$ personnes ont utilisé des services de SMart pour se salarier. Cette croissance a accompagné la transformation en entreprise coopérative, entamée en 2014. Au bout d'une première phase de cette transformation, SMArt s'est organisée en trois entités juridiques : Formateurs Associés, Palais de l'Intérim, Productions Associées qui, en 2018, réalisent un chiffre d'affaires total de presque 156 millions d'euros. La logique d'action de SMArt relève essentiellement du type "action syndicale élargie », mais dans une perspective qui la dépasse de plus en plus, car il s'agit de créer une entreprise nouvelle et de lui donner une dimension internationale. SMArt a investi et développé son concept dans plusieurs pays européens, à tel point qu'aujourd'hui elle peut être considérée comme la première «Firme multinationale de Travailleurs Autonomes Salariés ».

À la différence de Coopaname qui, au titre de l'insertion, bénéficie de financements publics, le modèle économique de SMArt et de Doc Servizi est fondé uniquement sur l'activité de ses membres et sur l'activité propre de la structure.

Tout comme Coopaname, SMArt ne refuse que l'une des caractéristiques du salariat : le lien de subordination. En revanche, elle vise à valoriser les formes du salaire socialisé, notamment la protection sociale. Critique vis-à-vis des expériences d'auto-gestion, SMArt a promu une forme d'entreprise traditionnelle, hiérarchique, pour ce qui est de l'organisation des pouvoirs. Néanmoins, avec son « devenir coopérative », un changement s'amorce. Si l'on s'en tient à son lexique, SMArt se définit maintenant comme une "entreprise partagée », ce qui la rapproche de Coopaname, en marquant la volonté de partager le pouvoir de décision à tous les niveaux de l'organisation. Pour comprendre cette convergence, il faut prendre en compte le fait que Sandrino Graceffa, nommé à la tête de SMArt au milieu des années 2010 pour conduire le changement, vient du monde des CAE et que, à cette même époque, SMArt, notamment SMArt.Fr, Cooopaname et Oxalis - une autre CAE - se sont rapprochées pour créer une structure commune : BIGRE!, sorte de «coentreprise » (joint-venture en anglais) visant la construction de «mutuelles de travail », projet pensé initialement par Coopaname. Ces rapprochements favorisent les échanges d'expériences et d'idées et préfigurent un possible devenir commun, même si la grande taille de SMArt peut constituer un frein à l'objectif politique de distribution des pouvoirs promu par Coopaname.

Le niveau politique de l'action collective de Coopaname a influencé aussi l'évolution de Doc Servizi depuis 2015, lorsqu'il y a eu la première rencontre des deux coopératives. Tout comme SMArt et Coopaname, Doc Servizi a connu une forte croissance ${ }^{6}$. Elle a été pionnière dans l'organisation de plateformes digitales. Le modèle de Doc Servizi est celui de la plateforme coopérative d'autogestion. La plateforme vise à recomposer le collectif de travail. C'est aussi un support important pour la recherche de clients et pour le développement de projets. La philosophie qui inspire Doc Servizi est similaire à celle de Coopaname. De même, il y a des similitudes, sinon dans les outils qui sont forgés à partir des institutions nationales, dans les objectifs poursuivis. Une différence majeure tient au fait que Doc Servizi, contrairement à Coopaname, est organisée autour des seules activités artistiques et culturelles. En croisant l'expérience de Coopaname, Doc Servizi a entamé un processus de diversification, en s'ouvrant à de nouvelles figures du travail indépendant en solo et qualifié. La rencontre avec l'expérience française des CAE a incité Doc Servizi à envisager une stratégie pour faire reconnaître le statut d'entrepreneur-salarié-associé, au lieu de poursuivre une stratégie de «bidouillage». Doc Servizi se constitue aussi en quasi-syndicat. Elle a œuvré, au niveau national, pour la reconnaissance du statut des travailleurs du spectacle des coopératives. Elle intervient donc aujourd'hui aux trois niveaux de l'action collective, tels qu'identifiés dans le schéma de D’Amours (2010). 


\section{Conclusion}

Les I-Pros - nouveaux travailleurs indépendants qualifiés — ont fait l'objet dans cet article d'une investigation en trois étapes, à la focale de plus en plus resserrée. La comparaison des contextes nationaux en Belgique, France et Italie a fait ressortir les nouvelles formes de précarité, propres aux figures du travail dans les zones grises, par-delà les différences des contextes institutionnels nationaux. D'une part, des droits moindres et/ou les difficultés d'accès aux droits sociaux; d'autre part, la discontinuité et l'imprévisibilité des contrats et des revenus. Face à ce défi, ont été caractérisées les logiques d'action de divers collectifs dont l'originalité est souvent d'intervenir dans une multiplicité de domaines. Ont été enfin étudiées les trajectoires de trois entreprises d'entrepreneuriat coopératif.

Il ressort plusieurs résultats.

Le premier est l'originalité de la logique coopérative comme mode d'action collective des I-Pros. Elle repose en effet sur des interventions multiples et différenciées sur un spectre étendu de règles de droit (des règles commerciales au droit social). Plus important encore, la logique coopérative transforme le travailleur indépendant en une figure inédite de l'histoire du travail, le travailleur salarié autonome.

Le deuxième est l'intensité de l'action menée par les trois coopératives observées, action qui passe d'abord par l'adaptation ou le détournement des règles instituées, puis par la révision des règles de droit (action de lobbying) mais aussi par la fabrication de nouveaux imaginaires, comme l'idée d'entreprise partagée ou de mutuelle de travail.

En effet, l'effort pour dé-précariser les nouveaux travailleurs autonomes ne se limite pas à l'action sur les règles de la protection sociale, il conduit à la réinvention de l'entreprise, en tant qu'institution. Dans l'aventure française des CAE, avec Coopaname, la rhétorique initiale du tremplin offert aux porteurs de projets créateurs de leur emploi a progressivement fait place à une ambition plus grande : dépasser l'alternative entre travail salarié subordonné et travail indépendant précaire.

La mise en regard des évolutions des trois expériences permet de dessiner une ligne de convergence autour de l'institution de «l'entrepreneur--salarié-associé », figure du travailleur autonome et interdépendant. Certes, comme cela a été bien observé en analysant le cas de SMart, le processus instituant est loin d'être achevé, car le mode de fonctionnement expérimental confère aux règles internes une instabilité récurrente et les principes de démocratie formelle qui fondent les entreprises coopératives ne suffisent pas à garantir la démocratie réelle (Charles, Ferreras et Lamine, 2019). Notons, à cet égard, que la sémantique des trois coopératives est différente: alors que Doc Servizi et SMArt insistent sur l'entrepreneuriat, le professionnalisme, Coopaname opte tout simplement pour le mot " coopérant", ce que l'on peut interpréter comme une volonté de signifier la coopération avant tout. Et ses Rapports d'activités mettent l'accent sur les dispositifs de démocratie, de partage et de diffusion du pouvoir. Si Coopaname a privilégié le modèle politique d'entreprise, les deux autres ont davantage investi dans la cohérence et l'efficacité du modèle économique. On peut alors avancer l'hypothèse que le devenir de l'entrepreneuriat coopératif en Europe comme alternative radicale au travail salarié et au développement de l'emploi indépendant précaire reposera dans la capacité qu'auront ces nouvelles formes d'entreprises coopératives à articuler modèle politique et modèle économique. 


\section{Références bibliographiques}

Beuker, Laura, Frédéric Naedenoen et François Pichault (2019) «Belgian Country Case Study », dans Renata Semenza et François Pichault (dir.), The Challenges of Self-Employment in Europe. Status, Social Protection and Collective Representation, Cheltenham: Edward Elgar, p.78-84.

Bureau, Marie-Christine, Antonella Corsani, Olivier Giraud et Frédéric Rey, dir. (2019) Les zones grises des relations de travail et d'emploi. Un dictionnaire sociologique, Buenos Aires: TeseoPress.

Bureau, Marie-Christine, Antonella Corsani et Bernard Gazier (2019) «Country Case Study: France », dans R. Semenza et F. Pichault (dir.), The Challenges of Self-Employment in Europe. Status, Social Protection and Collective Representation, Cheltenham: Edward Elgar, p.84-92.

Bost, Élisabeth (2011) Aux Entreprenants Associés. La Coopérative d'activité et d'emploi, Valence : Éditions Repas.

Bureau, Marie-Christine et Antonella Corsani (2018) « Fabriques instituantes », dans M.C. Bureau, A. Corsani, O. Giraud et F. Rey (dir.), Dictionnaire des zones grises du travail et de l'emploi, Buenos Aires : TeseoPress, p. 139-148.

Castoriadis, Cornelius (1975) L'institution imaginaire de la société, Paris : Seuil, Essais.

Charles, Julien, Isabelle Ferreras, Auriane Lamine et al. (2019), SMart comme capacité collective. Usages d'une Coopérative de travailleurs autonomes. En ligne: $<\underline{\text { http://hdl.handle.net/2078.1/227026>. }}$

D’Amours, Martine (2010) «Les logiques d'action collective d'associations regroupant des travailleurs indépendants », Relations industrielles/Industrial Relations, 65 (2), 257-280.

Dupuis, Yves et Françoise Larré (1998) «Entre salariat et travail indépendant : les formes hybrides de mobilisation du travail », Travail et Emploi, 77, 1-14.

Dupuy, Camille (2014) «Ordre ou syndicat : Le Syndicat national des journalistes, entre régulation corporatiste et défense du salariat », Terrains et Travaux, 25 (2), 113-129.

Frison-Roche, Marie-Anne (2002) «Le besoin conjoint d'une régulation analogue des relations sociales et des marchés globalisés », Revue internationale de Droit Économique, XVI (1), 67-82.

Graceffa, Sandrino (2016) Refaire le monde du travail. Une alternative à l'Uberisation de l'économie, Valence : Éditions Repas.

Mahoney, James et Kathleen Thelen (2010) "A Theory of Gradual Institutional Change", dans J. Mahoney et K. Thelen (dir.), Explaining Institutional Change : Ambiguity, Agency and Power, Cambridge ${ }^{\circ}$ Cambridge University Press, p. 1-37. 
Martinelli, Francesca (2016) Autonomie professionnelle, entrepreneuriat et coopération. Le cas des Coopératives d'activités et d'emploi en France, Thèse de doctorat, Université de Bergame.

Mori, Anna et Bart Koene (2019) «Continuity and Discontinuity in collective Representation », dans R. Semenza et F. Pichault (dir.) The Challenges of Self-Employment in Europe. Status, Social Protection and Collective Representation, Cheltenham ${ }^{\circ}$ : Edward Elgar, p. $178-205$.

Nicolas Le Strat, Pascal (2016) Le travail du commun, Saint Germain sur Ille : Éditions du Commun.

Poncin, Béatrice (2004) Salariés sans patron ?, Vulaines sur Seine: Éditions du Croquant.

Rapelli, Stefano (2012) European I-Pros ${ }^{\circ}$ : A Study, London: Professional Contractors Group Ltd.

Reynaud, Jean-Daniel (1997) Les règles du jeu. L'action collective et la régulation sociale, Paris : A Colin, coll. «U », 1 ère édition 1989, 314 pages.

Roux, Nicolas (2019, « Matermittentes », dans M.-C. Bureau, A. Corsani, O. Giraud et F. Rey (dir.), Dictionnaire des zones grises du travail et de l'emploi, Buenos Aires : TeseoPress, p. 325-333.

Semenza, Renata et Anna Mori (2019) «Country Case Study: Italy », dans R. Semenza et F. Pichault (dir.), The Challenges of Self-Employment in Europe. Status, Social Protection and Collective Representation, Cheltenhamº: Edward Elgar, p. 99-105.

Semenza, Renata et François Pichault, dir. (2019) The Challenges of Self-Employment in Europe. Status, Social Protection and Collective Representation, Cheltenham ${ }^{\circ}$ : Edward Elgar, 256 pages.

(de) Terssac, Gilbert (2012) «La théorie de la régulation sociale : repères introductifs », Revue Interventions économiques, 45 [En ligne].

\section{Documents consultés}

Coopaname (2019) Rapport d'activités 2018, Coopaname, France. 
DOC Servizi (2017) Bilancio Sociale 1990- 2017. Una lunga storia di impegno per l'arte, DOC Servizi, Italie.

Grelet-Certenais, N. (2020) «Rapport fait au nom de la Commission des Affaires Sociales sur la proposition de loi visant à rétablir les droits sociaux des travailleurs numériques », Sénat, 8 janvier.

SMArt (2019) Rapport d'activités 2018, Perspectives 2019 Belgique-France, SMArt, Belgique.

\section{Notes}

${ }^{1}$ URL : <https://www.i-wire.eu/>, consulté le 02-02-2020.

${ }^{2}$ Doc Servizi n'avait pas été retenu par l'équipe italienne d'I-WIRE, mais l'accès à un travail doctoral comparant Coopaname et Doc Servizi (Martinelli, 2016) nous a convaincus de l'intérêt de sa prise en compte.

${ }^{3}$ Il faut rappeler qu'à cette époque il n'existait pas en Belgique de statut de l'artiste. En France, les artistes du spectacle sont présumés salariés depuis 1969. Les artistes plasticiens relèvent de la Maison des Artistes. Créée en 1952 par des artistes plasticiens, la «Maison des Artistes » vise à rassembler, favoriser l'entraide et solliciter la réflexion pour améliorer la condition professionnelle des artistes (https://www.lamaisondesartistes.fr/).

${ }^{4}$ Extraits du récit de la rencontre Coopaname-Doc Servizi 2015 à laquelle nous avons participé dans le cadre de notre recherche sur les CAE. Cette rencontre a constitué le premier moment constitutif d'une coopération entre coopératives. En 2018, de nouvelles rencontres ont eu lieu entre Coopaname et Doc Servizi auxquelles ont été aussi conviées les coopératives Calidoscoop en Espagne, Comit en Allemagne et Neomania en Suisse. Ces coopératives envisagent la constitution au niveau européen d'un réseau de coopératives de travailleurs autonomes.

${ }^{5}$ Exemple tiré d'un entretien avec Sandrino Graceffa, dirigeant de SMArt, réalisé le 14 mai 2014.

${ }^{6}$ Voici quelques chiffres tirés du Rapport d'activités 2019: en 2018, Doc Servizi réalise un chiffre d'affaires de 50,5 millions, elle compte plus de 2 millions de journées de travail, $6^{\circ} 388$ membres associés, $4^{\circ} 200$ heures de formation pour ses salariés et 115 appels d'offres gagnés.

\section{RÉSUMÉ}

\section{Coopératives de travailleurs autonomes : du jeu dans les règles au jeu sur les règles, éléments de comparaison Belgique, France et Italie}

La situation et l'activité de nouveaux travailleurs indépendants qualifiés («I-Pros ») en Belgique, France et Italie font l'objet dans cet article d'une investigation en trois étapes, à la focale de plus en plus resserrée. Tout d'abord, une comparaison des contextes nationaux fait ressortir leur inconfort et leur précarité au sein d'une «zone grise » entre salariat et travail indépendant. Face à ce défi, l'article explore, ensuite, les logiques d'action de divers collectifs de travailleurs dont l'originalité est souvent d'intervenir simultanément dans une multiplicité de domaines. On détaille, enfin, les trajectoires de trois entreprises d'entrepreneuriat coopératif, chacune dans un des pays retenus. 
Prolongeant une recherche internationale sur l'organisation des travailleurs indépendants en Europe (Recherche «I-Wire »), l'article utilise les données et les analyses obtenues dans ce cadre et les complète tant sur le plan théorique (en mobilisant le concept de «travail instituant») que sur le plan empirique (en enrichissant le corpus dans le domaine des coopératives de travailleurs autonomes). La méthode adoptée combine 1 'analyse des institutions et organisations, et les interviews individuelles et collectives d'acteurs.

De ces premiers repérages au sein d'un paysage complexe et mouvant, il ressort un résultat principal : les « I-pros » sont souvent au cœur d'un jeu simultanément dans les règles et sur les règles. Notamment, les travailleurs des trois coopératives examinées sont engagés dans un intense «travail instituant» qui passe par la révision des règles de droit, mais aussi par la fabrication de nouveaux imaginaires. Les trajectoires fortement convergentes, mais néanmoins distinctes, des trois organisations permettent de déceler des référentiels à dominante économique, qui valorisent les projets professionnels et l'entrepreneuriat, et des référentiels à dominante politique, qui insistent sur l'idée de coopération citoyenne.

Mots-clés : travail indépendant, autonomie, coopératives d'activités et d'emploi, salarié contractuel, précarité.

\section{SUMMARY}

\section{Cooperatives of Self-Employed Workers: From Playing within the Rules to Playing with the Rules. Elements for Comparison from Belgium, France and Italy}

This article is an investigation into the situation and activity of an emerging group of selfemployed skilled workers (called "I-Pros") in Belgium, France and Italy. The focus is initially broad: the analysis begins with a brief comparison of the national contexts of self-employment and the existing challenges for these workers in the three countries. Next, the focus is narrowed, and the authors identify and discuss the various forms of logic that underlie the action of several organizations that protect and represent "I-Pros." Finally, the analysis deepens with a study of the trajectories and strategies of three worker cooperatives: one from each of the countries under study.

The article builds on previous European research: the I-WIRE project, which was centred on the needs and social protection of the self-employed in Europe. Here, its results are used and enriched on the theoretical level (through the concept of "instituting work") and on the empirical level (by gathering additional material and making additional interviews). The method involves combining analysis of institutions and organizations with individual or group interviews of relevant individuals.

The research remains exploratory because its object of study is complex and changing. However, one main result can be identified: the "I-pros" often play simultaneously within the rules and with the rules. In particular, the workers at the three cooperatives under study are engaged in an intense effort to "institute" their work not only by changing the legal rules but also by imagining new ways of being and doing. The three organizations have strongly convergent but nonetheless distinct trajectories. It is thus possible to distinguish between primarily economic frames of reference, which emphasize career plans and entrepreneurship, and primarily political ones, which stress the idea of citizen cooperation.

Keywords: self-employment, autonomy, Cooperatives of Activities and Employment, contract worker, job insecurity. 